\title{
A Geometric Fuzzy-Based Approach for Airport Clustering
}

\author{
Maria Nadia Postorino and Mario Versaci \\ Department of Civil Engineering, Energy, Environment and Materials, Mediterranea University of Reggio Calabria, \\ 89122 Reggio Calabria, Italy \\ Correspondence should be addressed to Mario Versaci; mario.versaci@unirc.it
}

Received 11 March 2014; Accepted 19 May 2014; Published 17 July 2014

Academic Editor: Francesco Carlo Morabito

Copyright ( $) 2014$ M. N. Postorino and M. Versaci. This is an open access article distributed under the Creative Commons Attribution License, which permits unrestricted use, distribution, and reproduction in any medium, provided the original work is properly cited.

\begin{abstract}
Airport classification is a common need in the air transport field due to several purposes-such as resource allocation, identification of crucial nodes, and real-time identification of substitute nodes-which also depend on the involved actors' expectations. In this paper a fuzzy-based procedure has been proposed to cluster airports by using a fuzzy geometric point of view according to the concept of unit-hypercube. By representing each airport as a point in the given reference metric space, the geometric distance among airports-which corresponds to a measure of similarity-has in fact an intrinsic fuzzy nature due to the airport specific characteristics. The proposed procedure has been applied to a test case concerning the Italian airport network and the obtained results are in line with expectations.
\end{abstract}

\section{Introduction}

Airports are crucial nodes of the air transport networks both as air terminals and as interchange nodes. As air terminals they represent a starting and ending point of flights. As interchange nodes they are the place where passengers transfer from one transport mode to another (surface/air and vice versa). The role of interchange nodes also depends on the existence of a well-developed surface network that links an airport to a given geographical region.

According to Eurocontrol figures [1], 170000 links of the European air traffic network rely on some 2000 airportsamong more than 2100-which can be considered fundamental nodes of the airport network. As stated in that report "understanding the variety of airports in Europe, their distribution, their traffic patterns, their aircraft mix, their strengths and their weaknesses is essential to understanding the strengths of the air traffic network as a whole."

The classification of elements is a common rule to identify some "types" according to specific goals. As an example, the above Eurocontrol report highlights the importance of "understanding the variety of airports" to understand the strengths of the whole air traffic network. Still in EU, four airport categories (community, national, large regional, and small regional) are identified (see [2]) with the specific aim to identify similar airports and particularly regional airports that are supposed to play an important role in supporting many Union policies [3].

Airports can be classified according to their size, functions, and ownership. As for size and functions, the International Civil Aviation Organisation (ICAO) provides classifications not only based on the geometric characteristics of both runways and aircraft but also based on the airport function measured by the airport traffic density [4]. Similar classifications are also made by the Federal Aviation Administration (FAA).

As for ownership, here the classification can be fainter due to different opportunities defined by specific laws at country level. However, according to a recent study by ICAO [5], autonomous airports are the most common form, accounting for $40 \%$ of the sampled airports $(80 \%$ of them state owned and the remaining privately owned). Governmental owned and/or managed airports and airports operated under a concession or leasing agreement represent the other two main groups while a further group includes other peculiar forms of ownership/management.

The identification of similar airports on the basis of some criteria and according to some specific goals can be used for 
various purposes. Although criteria and purposes can be very different, however, two important aims are (1) the identification of potential substitute nodes in the air network and (2) the identification of crucial nodes in the airport network to invest or allocate resources. In the first case, also real time features may be relevant. For example if unexpected events such as volcanic eruptions or severe meteorological conditions prevent using one or more airports, potential substitute nodes having similar characteristics should be identified in very short time. In the second case, uncertainty aspects may play a significant role because, whatever the classification procedures are, one airport cannot be considered absolutely similar to another one but only similar to a given extent.

In the literature some studies dealt with airport classification to select categories with comparable passenger terminal systems [6], to examine alternative slot allocation strategies [7] or operational efficiency [8], to study the evolution of the European aviation network [9], to identify strategic groups sharing common attributes/roles, or to identify airport rankings $[10,11]$.

In the above works traditional clustering techniques have been used; however, they have a high computational complexity and are unsuitable for real time applications. Furthermore, they do not fully consider imprecision due to the inherent difficulty in gathering entities that differ among them because of the context and the peculiarity of each of them, independently of the data used to identify their similarities.

The goal of this paper is to propose a general procedure to cluster airports-according to one or more factors measuring their characteristics-by using a fuzzy approach [1214]. In fact, if real time and imprecision features represent key factors, fuzzy systems could help to identify the better methodology with short computing time [15-17].

The common characteristics of groups of airports have to be set, but the features of each airport have to be defined so as to verify which group it belongs to. This problem can be defined as a classification issue where the key factor is the distance from the airport cluster centres. Here the classification problem is in fact defined from a fuzzy geometric point of view where each airport is represented by a fuzzy set depending on some parameters. The fuzzy nature of the problem, however, is not identified in the airport in itself, but mainly in the distance among similar airports. In other words, similarities among airports can be measured by a distance that have an intrinsic fuzzy nature. In fact, each airport has different characteristics and can develop different levels, which make it a unique entity. Then, the similarity measure among such entities is not a crisp value.

The fuzzy cluster approach proposed here, as alternative method with respect to other crisp approaches, is based on the potentiality it can offer when two aspects of fuzziness are considered. The first one concerns the identification of an airport as a fuzzy set; then not only numerical values but also linguistic variables can be used to describe it. The second aspect concerns the distance-considered as fuzzy quantitythat measures the similarity between couples of airports.
Finally, it is worthwhile to note that the goal of this paper is not to discuss the implications of clusters obtained by using one or more specific criteria, but to set the fuzzy procedure and then test it on a real case. However, since different criteria can give very different clusters, some of the most relevant criteria are briefly described in the next section in order to give an overview according to several points of view.

The paper is organized as follows. Section 2 and its subsections describe the role of airports and the way to deal with it, an overview of the proposed fuzzy geometric approach, developed in terms of fuzzy subsethood operator, and its formalization for the examined problem. Section 3 describes the results obtained on a test case and Section 4 summarizes some conclusions.

\section{Materials and Methods}

2.1. Airport Roles and Clustering Criteria. In densely populated areas such as the EU, many airports are located at a relatively short distance among them. Particularly, regional airports are often close to each other and are faced with either cooperation/integration or competition strategies among them. Hubs or community airports too are not exempt from this challenge and the evolution of airport networks is also an indirect effect of different strategies $[18,19]$.

In these situations, classifications are important to identify similar airports from some points of view. Generally speaking, airports are complex entities due to the several involved actors, whose needs and expectations could be different. The interaction among actors produces the airport outcome, often identified as the number of yearly handled passengers or movements.

Travellers and airlines are two important actors and also users of the airport managed by an airport operator. According to the distinction between landside and airside, airport services and facilities for travellers and airlines have to be distinguished (Figure 1).

For travellers, services mainly refer to (i) airport-related services (e.g., waiting time to check-in and for security controls, baggage delivery, airport commercial activities, and car parking area availability), (ii) services offered as the result of airport operators and local authorities/transport companies agreements (e.g., bus/rail services from/to main cities), and (iii) services resulting from the interaction between airport operators and airlines (e.g., served destinations, flight frequency, and hub connection). For airlines, services mainly refer to (i) navigation aid services (e.g., ILS, VOR) and (ii) handling (e.g., refuelling, cabin cleaning, and baggage services among the most relevant services).

Finally, facilities mainly refer to parking areas and passenger terminal (landside) and runways, taxiway, and aprons (airside).

While the list above cannot be considered complete, however, there are several points of view to cluster airports on the basis of some criteria such as level-of-service variables, travellers' preferences, and airport facilities.

Travellers perceive similar airports according to their travel experiences. The main key factors are level-of-service 


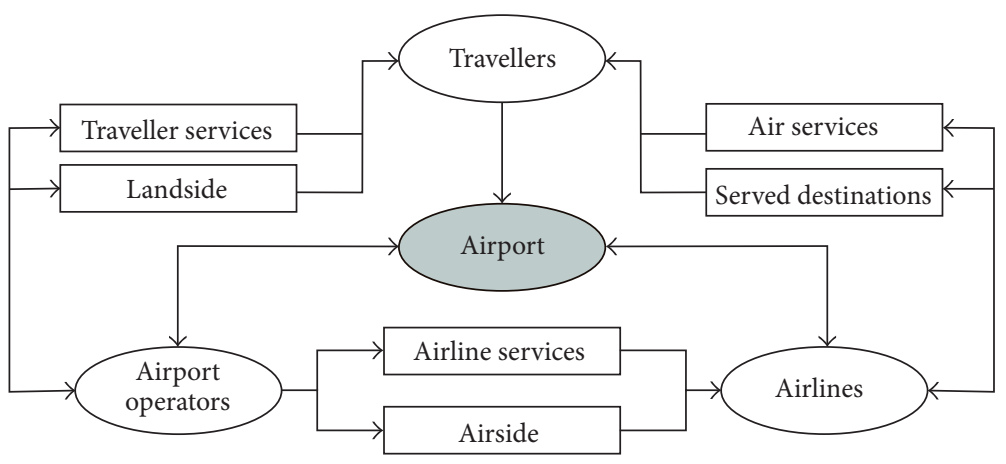

FIGURE 1: Relationships linking airport operators, airlines, and travellers with respect to the airport.

variables as described before, involving both airports and airlines and services offered by local transport companies. Clusters based on travellers' preferences can be useful for airport development policies in competitive markets. Furthermore, public planners-as governments and local authorities - may also represent the travellers' point of view to some extent, because they guarantee social wellness and the interests of their communities. In this light, they are interested in classifying and ranking airports to identify national/international airport network strategic nodes that guarantee accessibility to people also living in decentralized regions (EU, TEN-T Policy review). Finally, airlines choose airports for their network according to the services and facilities they offer with respect to their fleet composition requirements, the network type (e.g., hub-and-spoke versus point-to-point types), and the expected travel demand in the airport catchment areas [20, 21].

The key factors to identify similar airports are then different according to the point of view although in some cases they may lead to similar clusters. As an example, communityairport clusters obtained on the basis of the yearly passenger traffic-more than 10 million according to the EU [2] probably correspond to hub-airport clusters where services and facilities are the discriminating factors.

To express formally the problem, the vector $\mathbf{X}_{i}\left(\mathbf{X}_{i}=\right.$ $\left.\left\{x_{i, j}\right\}\right)$ characterizes the airport $i$ with respect to a given point of view so that the performances of $i$ are represented by $A_{i}=A\left(\mathbf{X}_{i}\right)$, where $A\left(\mathbf{X}_{i}\right)$ is a scalar function of some characteristics $\mathbf{X}_{i}$ defined in $\mathfrak{R}$. In the simplest case $\mathbf{X}_{i}$ is a $1 \times 1$ vector and corresponds to a single key factor.

Classifications can be realized in two ways: (1) by fixing the maximum number of clusters, $K$, that have to be identified according to some compulsory classes or identified categories (e.g., as in official classifications by ICAO and EU) and (2) without fixing the maximum number of classes that can be obtained.

In both cases, each cluster should gather "similar" entities whose membership could not be unique if they lie on the cluster frontier. There are some reasons that make the use of fuzzy approaches attractive as alternative methods with respect to crisp ones. First, "similarity" between airports does not correspond to "identity" and then the problem can be well represented by using a fuzzy approach, particularly a geometric one where "similarity" is translated in terms of distance in a certain space. Shortly, by using fuzzy logic each airport can be thought of as a fuzzy set. Further, a fuzzy set-and then an airport-can be represented by a point in a given $n$-dimensional metric space-not necessarily Euclideanwhere $n$ is the number of features extractable from an airport. "Similarities" among airports are assessable by distances among points. For a given couple of airports, the more the distance between them, the more the differences and vice versa. Generally, each airport has some specific characteristics that make it a unique entity. Furthermore, most of the airport characteristics have not fixed reference threshold values and they may vary within some undefined limits. Then, distance measures vary continuously in the given space for each couple of airports and can be identified by a fuzzy quantity represented by fuzzy values. Finally, while the membership of airports close to each other-and then close to the cluster centre-is clear, the same cannot be said for airports lying on the frontier and whose membership is more uncertain. The fuzzy approach can well represent such situations.

The next section describes the mathematical aspect of the fuzzy geometric approach applied to cluster similar entities.

\subsection{Geometric Point of View of Fuzzy Classification Prob-} lem. It is known that a fuzzy set $A$ can be considered as an abstract quantity containing other ones. Membership functions, which characterize a fuzzy set, are considered the kernel of mapping between objects and point elements belonging to $[0,1]$. However, in another perspective a fuzzy set can be viewed from a geometric point of view. In other words, a fuzzy set $A$ can be considered a point in a given space (Figure 2) whose metric is defined as

$$
d s^{2}=g_{i k} d x^{i} d x^{k}
$$

$g_{i k}$ being the so-called metrical tensor. Then, in the given space the distance among points can be calculated as

$$
\begin{gathered}
d s=\sqrt{g_{i k} d x^{i} d x^{k}}, \\
s=\int d s=\int \sqrt{g_{i k} d x^{i} d x^{k}} .
\end{gathered}
$$




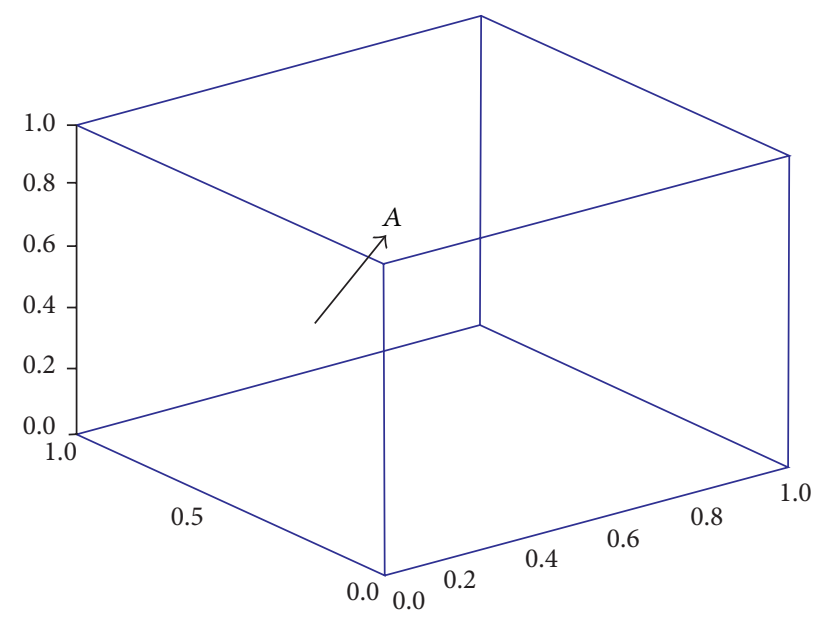

FIGURE 2: Representation of a fuzzy set $A$ within the unit-hypercube.

Since $d s^{2}$ is invariant as regards changes of the coordinate system-from $x^{i}$ to $x^{\prime i}$-such that $d s^{2}=d s^{\prime 2}$, then the $n$ dimensional Euclidean space $\mathfrak{R}^{n}$ can be used to compute distances. Since it occurs that

$$
g_{i k}=\delta_{i k}=\left(\begin{array}{ccc}
1 & \cdots & 0 \\
\vdots & \ddots & \vdots \\
0 & \cdots & 1
\end{array}\right),
$$

then (1) can be written as

$$
d s^{2}=\left(d x^{1}\right)^{2}+\left(d x^{2}\right)^{2}+\cdots+\left(d x^{n}\right)^{2} .
$$

By using the Einstein convention, (4) can also be written as

$$
d s^{2}=\left(d x^{i}\right)^{2}, \quad i=1, \ldots, n .
$$

Then, the distance between two fuzzy sets (or points) $A$ and $B, d(A, B)$, in the $n$-dimensional Euclidean space, is given by the length of the line connecting $A$ and $B$. When the problem under study is characterized by many variables, a fuzzy set can be thought of as a point inside a unit-hypercube in which each side is an unitary interval-since fuzzified point elements belong to $[0,1]$.

If $n$ is the number of variables, $2^{n}$ corners of the unithypercube represents crisp subsets, fuzzy subsets are located inside the unit-hypercube.

In particular, Cartesian coordinates of each point in the unit-hypercube are computed as fuzzified quantities $f^{A}\left(x_{j}\right)$. The geometric formulation of fuzzy sets, together with subsethood operators [22, 23], can play a crucial role as regards detection and classification problems. The basic idea is that a fuzzy set $A$ may be, to some extent, a subset of a fuzzy set $B$. When dealing with classification problems, subsethood operators can identify how much a fuzzy set $A$ is belonging to the class represented by the fuzzy set $B$ in the unit-hypercube. This geometric fuzzy approach has been already applied to some classification problems and compared with some other fuzzy clustering approaches [24]. However, it has not been applied in the field of transportation yet. In addition, with respect to canonical fuzzy approaches already tested in the literature the proposed geometrical approach is formulated in a space sized on features extracted directly from the airport characteristics, leading to the graphic translation of the clustering problem easily perceived even by nonexperts (points inside unit-hypercube). Obviously, appropriate choices of additional space (non-Euclidean) otherwise defined herein may help in the study of sets of airports with a high degree of overlap of features.

In this study, the points in the unit-hypercube are airports that have to be classified. The airport classification is obtained by identifying if and how much the fuzzy set $A$-unclassified airport-belongs to the fuzzy set $B$ representing a reference category of airports.

To describe the mathematical formulation, let $S(A, B)$ be the subsethood operator. $S(A, B)$ can be computed as follows:

$$
S(A, B)=1-\frac{\sum_{j=1}^{n} \max \left[0, f^{A}\left(x_{j}\right)-f^{B}\left(x_{j}\right)\right]}{M(A)},
$$

where $M(A)$ is the distance $d(A, B)$. Three types of distances are considered here:

(a) the Euclidean distance $d_{\text {euclidean }}(A, B)$ :

$$
M(A)^{2}=d_{\text {euclidean }}(A, B)^{2}=\left(x^{i}\right)^{2}=\left(f^{A}\left(x^{i}\right)-f^{B}\left(x^{i}\right)\right)^{2} ;
$$

(b) the fuzzy-Hamming distance $d_{\text {Hamming }}(A, B)$ :

$$
M(A)=d_{\text {Hamming }}(A, B)=\sum_{i=1}^{n} x^{i}=\sum_{i=1}^{n}\left|f^{A}\left(x^{i}\right)-f^{B}\left(x^{i}\right)\right| ;
$$

(c) the Kacprzyk distance $d_{\text {Kacprzyk }}(A, B)$ :

$$
M(A)=d_{\text {Kacprzyk }}(A, B)=\sum_{i=1}^{n} x^{i}=\sum_{i=1}^{n}\left|f^{A}\left(x^{i}\right)-f^{B}\left(x^{i}\right)\right|^{2} .
$$

It is easy to see that $S(A, B) \leq 1$ and $S(B, A)=1-S(A, B)$. Since $S(A, B)$ measures how much $A$ is contained in $B$, $S(B, A)$ measures how much $B$ is contained in $A$.

To summarize, reference categories of airports are points in the unit-hypercube and the positions of unclassified airports are identified as distance measures with respect to each known class of airports by using the subsethood operator. The following section explains in detail the classification procedure.

2.3. The Proposed Classification Procedure. According to the first classification criterion, that is, by fixing the maximum number of clusters, the basic idea of the proposed procedure starts from the consideration that the airport class $k$ is described by some parameters ranges. As showed by data, airports with fixed characteristics have parameters values 
(average, standard deviation, skewness, and kurtosis) falling into particular ranges. Then, for each class $k$, the average, standard deviation, skewness, and kurtosis values (labeled as $\mathrm{Av}^{k}, \mathrm{St}^{k}, \mathrm{Sk}^{k}$, and $\mathrm{Ku}^{k}$, resp.) are computed and the following tridimensional matrix $Z$ is obtained:

$$
Z=\left[\begin{array}{cccc}
\mathrm{Av}^{1}(j) & \mathrm{St}^{1}(j) & \mathrm{Sk}^{1}(j) & \mathrm{Ku}^{1}(j) \\
\cdots & \cdots & \cdots & \cdots \\
\cdots & \cdots & \cdots & \cdots \\
\operatorname{Av}^{k}(j) & \operatorname{St}^{k}(j) & \operatorname{Sk}^{k}(j) & \mathrm{Ku}^{k}(j)
\end{array}\right]
$$

The number of rows, $K$, is equal to the number of classes; the number of columns is equal to the number of parameters. The third dimension refers to the number of values available for each parameter and for each class $k$, for example, yearly or seasonal values. To describe the fuzzy clustering procedure when the number of classes is fixed a priori, $K=4$ has been chosen because it is the same number of classes adopted by the EU to classify airports as regards the yearly handled passengers. Note that the choice of $K=4$ must be considered only an example, although it refers to a real case, and it does not affect the generality of the procedure. Then the matrix $Z$ is specified as

$$
Z=\left[\begin{array}{llll}
\operatorname{Av}^{1}(j) & \mathrm{St}^{1}(j) & \mathrm{Sk}^{1}(j) & \mathrm{Ku}^{1}(j) \\
\operatorname{Av}^{2}(j) & \mathrm{St}^{2}(j) & \mathrm{Sk}^{2}(j) & \mathrm{Ku}^{2}(j) \\
\operatorname{Av}^{3}(j) & \mathrm{St}^{3}(j) & \operatorname{Sk}^{3}(j) & \mathrm{Ku}^{3}(j) \\
\operatorname{Av}^{4}(j) & \operatorname{St}^{4}(j) & \operatorname{Sk}^{4}(j) & \mathrm{Ku}^{4}(j)
\end{array}\right]
$$

Two matrices, $\min (Z)$ and $\max (Z)$, which represent, respectively, the matrices with the max and min values of the parameters, are also defined:

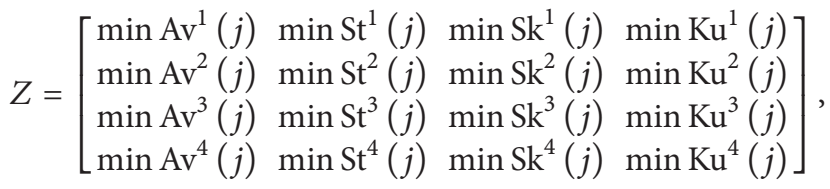

$$
\begin{aligned}
& Z=\left[\begin{array}{cccc}
\max \mathrm{Av}^{1}(j) & \max \mathrm{St}^{1}(j) & \max \mathrm{Sk}^{1}(j) & \max \mathrm{Ku}^{1}(j) \\
\max \mathrm{Av}^{2}(j) & \max _{\mathrm{St}^{2}(j)} & \max ^{2} \mathrm{Sk}^{2}(j) & \max \mathrm{Ku}^{2}(j) \\
\max \mathrm{Av}^{3}(j) & \max \mathrm{St}^{3}(j) & \max ^{3} \mathrm{Sk}^{3}(j) & \max \mathrm{Ku}^{3}(j) \\
\max \mathrm{Av}^{4}(j) & \max \mathrm{St}^{4}(j) & \max \mathrm{Sk}^{4}(j) & \max \mathrm{Ku}^{4}(j)
\end{array}\right] .
\end{aligned}
$$

Finally, the matrix $Z^{\prime}$, whose generic element is the interval $[\min (Z), \max (Z)]$, is computed as follows:

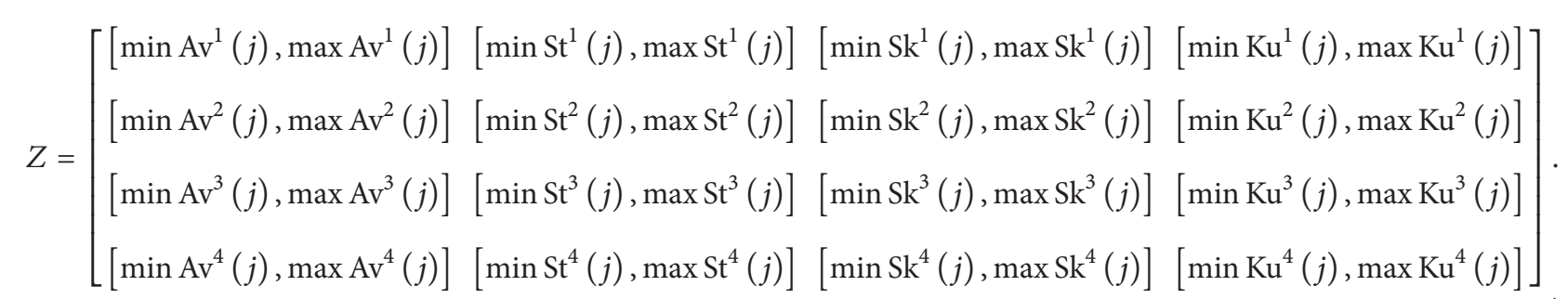

The fuzzification step leads to treating each element of $Z^{\prime}\left[\min \left(\operatorname{Statistical} \_\operatorname{Parameter}^{k}(j)\right), \quad \max \right.$ (Statistical_Parameter $\left.\left.{ }^{k}(j)\right)\right]$ by means of a suitable shaped function into the interval $[0,1]$. Here a sigmoid function has been chosen because of its very good smooth properties. However, other typologies of functions can also be considered.

Definitively, (14) shows the formulation of the fuzzification step. Each range of possible values of the statistical parameters is "translated" into a fuzzified range:

$$
\begin{aligned}
& f\left(Z^{\prime}(r, s)\right) \\
& \quad=\left[\frac{1}{1+e^{-Z^{k}\left(\inf \left(Z^{k}(r, s)\right)-H^{k}\right)}}, \frac{1}{1+e^{-Z^{k}\left(\sup \left(Z^{k}(r, s)\right)-H^{k}\right)}}\right],
\end{aligned}
$$

with $r, s$ generic row and column of matrix $Z^{\prime}$ and $S^{k}, H^{k}$ suitable sigmoidal parameters (referred to as each $k$ th airport class) located as a fuzzy range inside the 4-dimensional unithypercube.

If some satellite subclasses belong to a given class $k$, then the subclasses have to be included into the macroclass representing all the others. Then, the fuzzified range is computed as

$$
\begin{aligned}
& {\left[\min _{\text {sub-classes }}(f(\min (Z(r, s)))),\right.} \\
& \left.\max _{\text {sub-classes }}(f(\min (Z(r, s))))\right] .
\end{aligned}
$$

If $Y$ is a new airport that has to be classified, the vector $\mathbf{Y}$ of its parameters is

$$
\mathbf{Y}=\left\lfloor\mathrm{Av}^{k}, \mathrm{St}^{k}, \mathrm{Sk}^{k}, \mathrm{Ku}^{k}\right\rfloor
$$

and it is fuzzified by using a sigmoid function as

$$
f(\mathbf{Y}(h))=\frac{1}{1+e^{-s(\mathbf{Y}(h))}}, \quad h=1,2,3,4,
$$

where $f(\mathbf{Y}(h))$ is a point inside the 4-dimensional unithypercube. Then, the quantity,

$$
S\left(f(\mathbf{Y}(h)), f\left(\max _{\text {sub_classes }}(f(\min (Z(r, s))))\right)\right),
$$


TABLE 1: Commercial airports in Italy (reference year: 2012, source: http://www.assaeroporti.it/category/statistiche/).

\begin{tabular}{|c|c|c|c|c|c|}
\hline 1 Alghero, AHO & 2 Ancona, AOI & 3 Bari, BRI & 4 Bergamo, BGY & 5 Bologna, BLQ & 6 Bolzano, BZO \\
\hline 7 Brescia, VBS & 8 Brindisi, BDS & 9 Cagliari, CAG & 10 Catania, CTA & 11 Crotone, CRV & 12 Cuneo, CUF \\
\hline 13 Firenze, FLR & 14 Foggia, FOG & 15 Forlì, FRL & 16 Genova, GOA & 17 Lamezia Terme, SUF & 18 Milano Linate, LIN \\
\hline $\begin{array}{l}19 \text { Milano } \\
\text { Malpensa, MXP }\end{array}$ & 20 Napoli, NAP & 21 Olbia, OLB & 22 Palermo, PMO & 23 Parma, PMF & 24 Perugia, PEG \\
\hline 25 Pescara, PSR & 26 Pisa, PSA & 27 Reggio Calabria, REG & 28 Rimini, RMI & 29 Roma Ciampino, CIA & 30 Roma Fiumicino, FCO \\
\hline 31 Siena, SAY & 32 Torino, TRN & 33 Trapani, TS & 34 Treviso, TSF & 35 Trieste, TRS & 36 Venezia, VCE \\
\hline 37 Verona, VRN & 38 Grosseto, GRS & & & & \\
\hline
\end{tabular}

is computed as explained in (3). If the above quantity is closer to unity, the new airport likely belongs to the class $k$ and

$$
\max _{k} S\left(f(\mathbf{Y}(h)), f\left(\max _{\text {sub_classes }}(f(\min (Z(r, s))))\right)\right)
$$

identifies the membership class. In this case there are three subsethood operators [25], each one defined by a different metric.

The same procedure can be used to identify clusters without fixing a priori their maximum numbers. In fact,

(1) If $Y$ is the first examined airport, its vector $Y$ of parameters is

$$
\mathbf{Y}=\left\lfloor\mathrm{Av}^{k}, \mathrm{St}^{k}, \mathrm{Sk}^{k}, \mathrm{Ku}^{k}\right\rfloor .
$$

$\mathrm{Y}$ is fuzzified by using a sigmoid function (17):

$$
f(\mathbf{Y}(h))=\frac{1}{1+e^{-s(\mathbf{Y}(h))}}, \quad h=1,2, \ldots, K
$$

and the airport $Y$ is a single point inside the unithypercube (cluster I).

(2) If $Y^{\prime}$ is the second examined airport, its vector $Y^{\prime}$ of parameters is

$$
\mathbf{Y}^{\prime}=\left\lfloor\mathrm{Av}^{k^{\prime}}, \mathrm{St}^{k^{\prime}}, \mathrm{Sk}^{k^{\prime}}, \mathrm{Ku}^{k^{\prime}}\right\rfloor .
$$

$\mathbf{Y}^{\prime}$ is fuzzified by using a sigmoid function (17):

$$
f\left(\mathbf{Y}^{\prime}(h)\right)=\frac{1}{1+e^{-s\left(\mathbf{Y}^{\prime}(h)\right)}}, \quad h=1,2, \ldots, K
$$

and the airport $Y^{\prime}$ is another point inside the unithypercube (cluster II).

(3) If $Y^{\prime \prime}$ is the third examined airport, its vector $\mathbf{Y}^{\prime \prime}$ of parameters is

$$
\mathbf{Y}^{\prime \prime}=\left\lfloor\mathrm{Av}^{k^{\prime \prime}}, \mathrm{St}^{k^{\prime \prime}}, \mathrm{Sk}^{k^{\prime \prime}}, \mathrm{Ku}^{k^{\prime \prime}}\right\rfloor .
$$

$\mathrm{Y}^{\prime \prime}$ is fuzzified by using a sigmoid function (17):

$f\left(\mathbf{Y}^{\prime \prime}(h)\right)=\frac{1}{1+e^{-s\left(\mathbf{Y}^{\prime \prime}(h)\right)}}, \quad h=1,2, \ldots, K$

and the airport $Y^{\prime \prime}$ is another point inside the unithypercube.
(4) The values of $S\left(Y^{\prime \prime}, Y\right)$ and $S\left(Y^{\prime}, Y\right)$ are then computed. If their values are greater than a prefixed threshold, a third cluster is identified; otherwise the minimum value between those ones identifies the airport membership to its class. The procedure continues until the last airport has been examined.

\section{Application to a Test Case}

The procedure described in the previous sections has been applied to the Italian airport system. According to the National Authority for Civil Aviation [26], in Italy there are 45 certified commercial airports. However, at year 2013 only 38 are included in the official figures (Table 1) because the others handle an insufficient number of passengers. In Table 1, the airports are identified with a numerical label, the name of the main city they serve, and the IATA code.

As discussed in Section 2, the criteria to classify airports can vary according to both the point of view and the goal to be achieved. It is worthwhile to note that the aim of the paper is to present and then test the airport clustering fuzzy geometric approach based on the concept of "similarity" as fuzzy distance rather than providing specific policy recommendations on the basis of the application to the test case. In this light, among the several and various criteria that can be identified, the ones considered here are based on data available at national level and provided by the Association of Italian Airports (Assaeroporti, http://www.assaeroporti.it/). The choice of such data to cluster airports has also been motivated by the current policy adopted by the Italian government to identify the relevant airports for the Italian airport network. Particularly, the official data used here as classification criteria refer to the yearly number of movements and handled passengers. The chosen criteria take into account the airport dimensions. If data are available, other criteria such as served destinations or airport connectivity could take into account the airport attractiveness.

According to the procedure described in Section 4, the airport is represented by a fuzzy set and then yearly passengers and movements values have been fuzzified (Table 2). The number in the first row corresponds to the airport numerical label as in Table 1 . The ranges reported in 
TABLE 2: Yearly ranges (15) for each airport coded as in Table 1.

\begin{tabular}{|c|c|c|}
\hline Airport & $\begin{array}{c}\text { Criterion } 1 \\
\text { movements }\end{array}$ & $\begin{array}{l}\text { Criterion } 2 \\
\text { passengers } \\
\end{array}$ \\
\hline 1 & $0-0.461$ & $0-0.642$ \\
\hline 2 & $0-0.481$ & $0-0.387$ \\
\hline 3 & $0-0.559$ & $0-0.544$ \\
\hline 4 & $0-0.388$ & $0-0.372$ \\
\hline 5 & $0-0.421$ & $0-0.439$ \\
\hline 6 & $0-0.313$ & $0-0.345$ \\
\hline 7 & $0-0.291$ & $0-0.309$ \\
\hline 8 & $0-0.297$ & $0-0.344$ \\
\hline 9 & $0-0.355$ & $0-0.390$ \\
\hline 10 & $0-0.581$ & $0-0.614$ \\
\hline 11 & $0-0.142$ & $0-0.134$ \\
\hline 12 & $0-0.172$ & $0-0.177$ \\
\hline 13 & $0-0.422$ & $0-0.429$ \\
\hline 14 & $0-0.296$ & $0-0.298$ \\
\hline 15 & $0-0.219$ & $0-0.245$ \\
\hline 16 & $0-0.381$ & $0-0.401$ \\
\hline 17 & $0-0.237$ & $0-0.249$ \\
\hline 18 & $0-0.538$ & $0-0.534$ \\
\hline 19 & $0-0.804$ & $0-0.810$ \\
\hline 20 & $0-0.632$ & $0-0.654$ \\
\hline 21 & $0-0.415$ & $0-0.390$ \\
\hline 22 & $0-0.458$ & $0-0.465$ \\
\hline 23 & $0-0.312$ & $0-0.341$ \\
\hline 24 & $0-0.311$ & $0-0.286$ \\
\hline 25 & $0-0.388$ & $0-0.386$ \\
\hline 26 & $0-0.387$ & $0-0.399$ \\
\hline 27 & $0-0.314$ & $0-0.387$ \\
\hline 28 & $0-0.465$ & $0-0.432$ \\
\hline 29 & $0-0.481$ & $0-0.511$ \\
\hline 30 & $0-0.801$ & $0-0.843$ \\
\hline 31 & $0-0.180$ & $0-0.208$ \\
\hline 32 & $0-0.625$ & $0-0.607$ \\
\hline 33 & $0-0.219$ & $0-0.218$ \\
\hline 34 & $0-0.230$ & $0-0.201$ \\
\hline 35 & $0-0.314$ & $0-0.318$ \\
\hline 36 & $0-0.514$ & $0-0.483$ \\
\hline 37 & $0-0.415$ & $0-0.429$ \\
\hline 38 & $0-0.172$ & $0-0.194$ \\
\hline
\end{tabular}

Table 2 have been obtained by using (15), here reported for clarity,

$$
\begin{array}{r}
\text { ranges }\left[\min _{\text {subclasses }}(f(\min >(Z(r, s)))),\right. \\
\left.\max _{\text {subclasses }}(f(\min (Z(r, s))))\right] .
\end{array}
$$

Although the used data could not be considered intrinsically fuzzy, however, other kinds of data could be such as travellers' preferences also expressed as linguistic variables or level-ofservice variables. As already stated, the goal of the paper is to test the proposed approach by using available data-in this case the ones available from the above official sources.

According to the general relationship $A_{i}=A\left(\mathbf{X}_{i}\right)$ described in Section 2, the experiments realized here refer to the simplest case where $\mathbf{X}_{i}$ is a single key factor (or criterion), particularly the yearly number of passengers and the yearly number of movements. 
TABLE 3: Clustering results with prefixed number of clusters. Criterion: yearly passengers.

\begin{tabular}{|c|c|c|c|}
\hline Metrics & ED & FHD & $\mathrm{KD}$ \\
\hline \multicolumn{4}{|c|}{ Cluster 1 (centre: Roma FCO) } \\
\hline$\leq 20 \%$ & $30,19,18,4,36$ & $30,19,18,4$ & $30,19,18,4$ \\
\hline $20 \%-40 \%$ & $10,20,29,5,22,26,9$ & $36,10,20,29,5,22,26$ & $36,10,20,29,5,22$ \\
\hline$>40 \%$ & $\begin{array}{l}32,37,3,34,21,13,17,1,16,8,35,33,15 \\
2,27,25,28,23,7,12,24,6,14,11,38,31\end{array}$ & $\begin{array}{c}9,32,37,3,34,21,13,17,1,16,8,35,33 \\
15,2,27,25,28,23,7,12,24,6,14,11,38 \\
31\end{array}$ & $\begin{array}{c}9,26,32,37,3,34,21,13,17,1,16,8,35, \\
33,15,2,27,25,28,23,7,12,24,6,14,11, \\
38,31\end{array}$ \\
\hline \multicolumn{4}{|c|}{ Cluster 2 (centre: Napoli Capodichino) } \\
\hline$\leq 20 \%$ & 20,10 & 20,10 & 20,10 \\
\hline $20 \%-40 \%$ & $9,32,36,37,4,26,36,3,29,22$ & $9,32,36,37,4,26,36,3,29,22$ & $9,32,36,37,4,26,36,3,29,22$ \\
\hline$>40 \%$ & $\begin{array}{c}18,30,19,26,5,21 \\
17,8,1,33,13,15,16,27,14,35,12,7,11 \\
2,28,31,23,38,24,6\end{array}$ & $\begin{array}{c}18,30,19,26,5 \\
21,17,8,1,33,13,15,16,27,14,35,12,7 \\
11,2,28,31,23,38,24,6\end{array}$ & $\begin{array}{c}18,30,19,26,5 \\
21,8,1,33,13,15,16,27,14,35,12,7,11 \\
2,28,31,23,38,24,6\end{array}$ \\
\hline \multicolumn{4}{|c|}{ Cluster 3 (centre: Lamezia Terme) } \\
\hline$\leq 20 \%$ & $17,8,34$ & $17,8,34$ & $17,8,34$ \\
\hline $20 \%-40 \%$ & $33,9,15,26,22,1$ & $33,9,15,26,22$ & $33,9,15,26,22$ \\
\hline$>40 \%$ & $\begin{array}{c}29,37,21,3,5,20,32,10,4,14,27,36,17 \\
18,30,19,7,12,13,11,25,16,31,38,35 \\
28,23,2,6,34\end{array}$ & $\begin{array}{c}1,29,37,21,3,5,20,32,10,4,14,27,36 \\
17,18,30,19,7,12,13,11,25,16,31,38 \\
35,28,23,2,6,34\end{array}$ & $\begin{array}{c}1,29,37,21,3,5,20,32,10,4,14,27,36 \\
17,18,30,19,7,12,13,11,25,16,31,38 \\
35,28,23,2,6,34\end{array}$ \\
\hline \multicolumn{4}{|c|}{ Cluster 4 (centre: Reggio Calabria) } \\
\hline$\leq 20 \%$ & $27,7,12$ & $27,7,12$ & $27,7,12$ \\
\hline $20 \%-40 \%$ & $25,11,15,31,38,14$ & $25,11,15,31,38$ & $25,11,15,31,38$ \\
\hline$>40 \%$ & $\begin{array}{c}33,16,13,8,17,28,23,35,34,26,9,22 \\
29,2,6,24,1,21,5,20,3,32,10,4,36 \\
18,30,19\end{array}$ & $\begin{array}{c}14,33,16,13,8,17,28,23,35,34,26,9 \\
22,29,2,6,24,1,21,5,20,3,32,10,4 \\
36,18,30,19\end{array}$ & $\begin{array}{c}14,33,16,13,8,17,28,23,35,34,26,9 \\
22,29,2,6,24,1,21,5,20,3,32,10,4 \\
36,18,30,19\end{array}$ \\
\hline
\end{tabular}

As regards the two classification procedures described in Section 4 , the airport clusters have been obtained by fixing the maximum number of classes (Tables 3 and 5) and without fixing the number of classes (Tables 4 and 6). Furthermore, the three distance metrics (Euclidean, ED; fuzzy-Hamming, FHD; and Kacprzyk, KD) have been used to identify the clusters.

In Tables 3 and 4, for each cluster and for each metric, the first list (in row) of airports refers to the closest airports as regards the cluster centre. Particularly, they are in the range of $20 \%$ of the max-min interval. The second list refers to those airports that are farther from the cluster centre and are in the range between $20 \%$ and $40 \%$ of the max-min interval. Finally, the third list refers to airports that are quite far from the centre and outside the range of $40 \%$ of the max-min interval.

From Tables 3-6 it can be seen that the three metrics provide rather similar results in terms of group membership although, as expected, some differences can be seen in terms of distance from the centre clusters. In fact, according to the metrics, the same elements may be farther or closer to the cluster centre but the group composition is practically identical.

Figure 3 summarizes the group composition with reference to passengers and movements with Euclidean distance (see Tables 3 and 5, first column) and provides an overview of the cluster overlaps.

For each cluster, identified by a circle, the two areas in each circle identify the first two subsets within the cluster
(Tables 3-6). In other words, they reproduce the airport lists as regards the distance from the centre $(\leq 20 \%$ of the max-min interval, between $20 \%$ and $40 \%$ of the max-min interval). The elements outside the circles represent those whose distance is greater than $40 \%$ of the max-min interval. The elements closest to the cluster centre are in the grey circle while the others are located inside the black circle (and outside the grey one) or outside the black circle according to their distance from the centre.

Elements in shared areas represent airports that could be located in more clusters according to a crisp distance threshold. In other words, the cluster membership obtained as a result of a fuzzy approach makes it possible to identify clustering uncertainty for elements farther from the centre.

The airports outside the black circles can be considered marginal within each cluster and significantly different from the cluster centre while the ones between the grey and black circles do not belong undoubtedly to the cluster but at the same time are not so different from the ones in the grey circle.

Shared areas among clusters are the consequence of the three subsets identified within each cluster. In fact, some airports can belong to different clusters with different membership values. This is one of the fuzzy approach advantages when grouping complex entities like airports. In fact, it is practically impossible to build homogeneous clusters of airports that have exactly the same characteristics or are just slightly different, but it is possible to find similarities to a certain extent. As regards the goal to identify the relevant 
TABLE 4: Clustering results without prefixing the number of clusters. Criterion: yearly passengers.

\begin{tabular}{|c|c|c|c|}
\hline Metrics & $\mathrm{ED}$ & FHD & $\mathrm{KD}$ \\
\hline \multicolumn{4}{|c|}{ Cluster 1 (centre: Roma FCO) } \\
\hline$\leq 20 \%$ & $30,19,18,36$ & $30,19,18,36$ & $30,19,18,36$ \\
\hline $20 \%-40 \%$ & $4,10,20,29,5,22,26,9$ & $4,10,20,29,5,22,9,26$ & $4,10,20,29,5,9,22,26$ \\
\hline$>40 \%$ & $\begin{array}{l}37,32,3,34,21,13,17,1,16,8,35,33,15 \\
2,27,25,28,23,7,12,24,6,14,11,31,38\end{array}$ & $\begin{array}{l}37,32,3,34,21,13,17,1,16,8,35,33,15, \\
2,27,25,28,23,7,12,24,6,14,11,38,31\end{array}$ & $\begin{array}{l}37,32,3,34,21,13,17,1,16,8,35,33,15 \\
27,2,25,28,23,7,12,24,6,14,38,11,31\end{array}$ \\
\hline
\end{tabular}

Cluster 2 (centre: Bologna)

\begin{tabular}{|c|c|c|c|}
\hline$\leq 20 \%$ & $5,29,22$ & $5,29,22$ & $5,29,22$ \\
\hline $20 \%-40 \%$ & $26,4,18,9,13,30,10,19,20$ & $26,4,18,9,13,30,10,20,19$ & $26,4,18,9,13,30,20,10,19$ \\
\hline$>40 \%$ & $\begin{array}{l}36,34,16,17,8,32,15,37,3,27,12,1,16 \\
21,7,25,35,14,12,28,2,23,31,38,24,6\end{array}$ & $\begin{array}{l}36,34,16,8,17,32,15,37,3,27,12,1,16 \\
21,7,25,35,12,14,28,2,23,31,38,6,24\end{array}$ & $\begin{array}{l}36,34,8,16,17,32,15,37,3,27,12,1,16 \\
21,25,7,35,12,14,28,2,23,31,6,38,24\end{array}$ \\
\hline
\end{tabular}

Cluster 3 (centre: Alghero)

$\leq 20 \%$

$20 \%-40 \%$

$>40 \%$

24

1

$21,34,37,3,17,8,32,20,9,15,10$

$36,26,29,4,22,18,15,14,30,19,5,11$, 24
1

$21,34,37,3,17,8,32,20,9,15$

$10,36,26,29,4,22,18,15,14,30,19,5$,

$11,12,27,7,13,16,25,31,35,38,28,23$, $2,24,6$
1

$21,34,37,3,17,8,32,20,9,15$

$10,36,29,26,4,22,18,15,30,14,19,5$, $11,12,27,7,13,16,25,31,35,38,28,23$, $24,2,6$

Cluster 4 (centre: Reggio Calabria)

\begin{tabular}{|c|c|c|c|}
\hline$\leq 20 \%$ & $27,7,12$ & $27,7,12$ & $27,7,12$ \\
\hline $20 \%-40 \%$ & $25,11,15,31,38,14$ & $25,11,15,31,14,38$ & $25,11,15,31,38,14$ \\
\hline$>40 \%$ & $\begin{array}{c}33,16,13,8,17,28,23,35,34,26,9,22 \\
29,2,6,24,1,21,5,20,3,32,10,4,36 \\
18,30,19\end{array}$ & $\begin{array}{c}16,33,13,8,17,28,23,35,34,26,9,22, \\
29,2,6,24,1,21,5,20,3,32,10,4,36 \\
18,19,30\end{array}$ & $\begin{array}{c}33,16,13,17,8,28,23,35,34,26,9,22 \\
29,2,6,24,1,21,5,20,3,32,10,4,18 \\
30,30,19\end{array}$ \\
\hline
\end{tabular}

Cluster 5 (centre: Siena)

$\leq 20 \%$

$20 \%-40 \%$

$>40 \%$
31

$38,7,12,11,25,27$

$14,15,28,6,24,23,33,2,16,8,13,17,35,27,14,15,28,6,24,23,33,2,16,8,13,17,14,15,28,24,6,23,33,2,16,8,13,17,35$, $34,26,22,5,1,9,29,20,21,37,3,32,10, \quad 35,34,26,5,22,1,9,20,29,21,37,3,32, \quad 34,26,5,22,1,9,20,29,21,37,3,32,10$, $4,18,36,30,19$
$10,4,18,36,19,30$

$4,18,19,36,30$ airports for the Italian airport network, airports in shared areas can be rightly examined within the national context by suitably taking into account their characteristics within one or more clusters.

As for the two classification procedures-with and without prefixed number of clusters-as expected the second one provides more homogeneous results in terms of the similarity of airports belonging to the same cluster. In fact, when the maximum number of clusters is not fixed the groups are formed by entities that are more homogeneous. At the extreme case, there are as many clusters as objects to be grouped; then each entity is considered different from the other ones.

The use of two clustering criteria (yearly passengers and movements) in this case provides similar results (Figure 3). In fact, generally there is a direct relationship between carried passengers and number of movements although in some situations the number of airport movements can also be due to other general aviation segments (e.g., military operations, cargo movements). Then, these results are in line with what is expected in principle because of the relationship between passengers and movements.

\section{Conclusions and Perspectives}

In this work, a fuzzy-based cluster procedure has been proposed to classify airports. Particularly, the concept of unithypercube has been used to recognize the airport cluster membership on the basis of some airport statistical parameters computed by using official data. As discussed, the choice of criteria depends on the classification goal and this is an important aspect. However, the paper focuses on the fuzzy clustering approach rather than on the choice of criteria; these latter depend on several points of view and have several policy implications. The main goal of the paper was in fact to test the fuzzy geometric approach as alternative method with respect to crisp ones rather than providing specific policy recommendations for airport selection or discussing classification implications by using different criteria. 
TABLE 5: Clustering results with prefixed number of clusters. Criterion: yearly movements.

Metrics

(a)

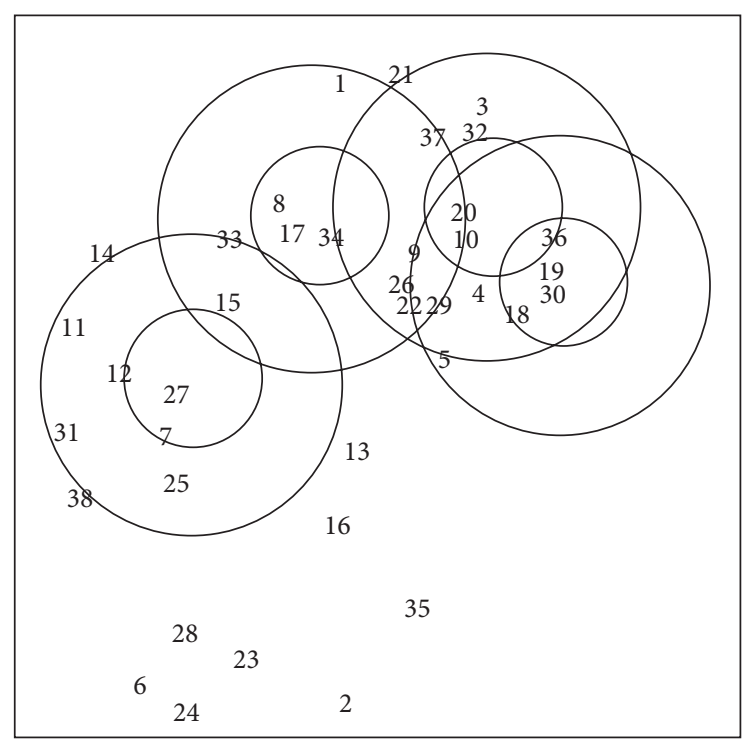

(b)

FIGURE 3: Representation of clusters (Tables 3 and 5-passengers and movements criteria with Euclidean distance).

According to the methodological features presented in the previous sections, the fuzzy procedure proposed here makes it possible to take into account uncertainty and imprecision of the similarity measures by using a geometric fuzzy representation. The fuzzy approach is here particularly desirable because of the nature of the problem. In fact, airports are complex elements and their potential clustering in crisp classification procedures is not as clear as it could appear, particularly when they lie on the cluster frontier. 
TABLE 6: Clustering results without prefixing the number of clusters. Criterion: yearly movements.

\begin{tabular}{|c|c|c|c|}
\hline Metrics & ED & FHD & $\mathrm{KD}$ \\
\hline \multicolumn{4}{|c|}{ Cluster 1 (centre: Roma FCO) } \\
\hline$\leq 20 \%$ & $30,19,18,36$ & $30,19,18,36$ & $30,19,18,36$ \\
\hline $20 \%-40 \%$ & $4,10,20,29,5,22$ & $4,10,20,29,5,22$ & $4,10,20,29,5,22$ \\
\hline$>40 \%$ & $\begin{array}{c}26,9,37,32,3,34,21,13,17,1,16,8,35 \\
33,15,2,27,28,25,23,7,12,24,6,14,11 \\
38,31\end{array}$ & $\begin{array}{c}26,9,37,32,3,34,21,13,17,1,16,8,35 \\
33,15,2,27,28,25,23,7,12,24,6,14 \\
38,11,31\end{array}$ & $\begin{array}{c}26,9,37,32,3,34,21,13,17,1,16,8,35 \\
33,15,2,27,28,25,23,7,12,24,6,14 \\
38,11,31\end{array}$ \\
\hline \multicolumn{4}{|c|}{ Cluster 2 (centre: Torino) } \\
\hline$\leq 20 \%$ & $32,3,37$ & $32,3,37$ & $32,3,37$ \\
\hline $20 \%-40 \%$ & $20,10,36,9,34,4$ & $20,10,36,9,34$ & $20,10,36,9,34$ \\
\hline$>40 \%$ & $\begin{array}{c}1,26,19,30,18,29,22,5,17,8,33,15,13 \\
16,27,14,16,12,7,11,25,35,31,38,28 \\
2,23,24,6\end{array}$ & $\begin{array}{c}4,1,26,19,18,30,29,22,5,17,8,33,15 \\
13,16,27,14,16,12,7,11,25,35,31,38 \\
28,2,23,6,24\end{array}$ & $\begin{array}{c}4,1,26,19,18,30,29,22,5,17,8,33,15 \\
13,16,14,27,16,12,7,11,25,35,31,38 \\
28,2,23,6,24\end{array}$ \\
\hline \multicolumn{4}{|c|}{ Cluster 3 (centre: Lamezia Terme) } \\
\hline$\leq 20 \%$ & $17,8,34$ & $17,8,34$ & $17,8,34$ \\
\hline $20 \%-40 \%$ & $33,9,15,26,22,1,29$ & $33,9,15,26,22,1,29$ & $33,9,15,26,22,1,29$ \\
\hline$>40 \%$ & $\begin{array}{c}21,37,3,5,20,32,10,4,14,27,36,17,30 \\
18,19,7,13,12,11,25,16,31,38,35,28 \\
23,2,6,34\end{array}$ & $\begin{array}{c}21,37,3,5,20,32,10,4,14,27,36,17,30 \\
18,19,7,13,12,11,25,16,38,31,35,28 \\
23,6,2,34\end{array}$ & $\begin{array}{c}21,37,5,3,20,32,10,4,14,27,36,17,30, \\
18,19,7,13,12,11,25,16,38,31,35,28, \\
23,2,6,34\end{array}$ \\
\hline \multicolumn{4}{|c|}{ Cluster 4 (centre: Pescara) } \\
\hline$\leq 20 \%$ & 25 & 25 & 25 \\
\hline $20 \%-40 \%$ & $7,27,38,12,31$ & $7,27,38,12,31$ & $7,27,38,12,31$ \\
\hline$>40 \%$ & $\begin{array}{c}28,16,11,23,13,6,14,15,24,2,33,35,8 \\
17,5,22,26,34,9,29,1,20,37,21,4,3, \\
32,10,18,30,36,19\end{array}$ & $\begin{array}{c}28,16,11,13,23,6,14,15,24,2,33,35,8 \\
17,5,22,26,9,34,29,1,20,37,21,4,3 \\
32,10,18,30,19,36\end{array}$ & $\begin{array}{c}28,16,11,13,23,6,14,15,24,2,33,35,8, \\
17,5,22,26,9,34,29,1,20,37,21,4,3, \\
32,10,30,18,19,36\end{array}$ \\
\hline \multicolumn{4}{|c|}{ Cluster 5 (centre: Crotone) } \\
\hline$\leq 20 \%$ & 11 & 11 & 11 \\
\hline $20 \%-40 \%$ & $32,14,31,7,27,15$ & $32,14,31,7,27,15$ & $32,14,31,7,27,15$ \\
\hline$>40 \%$ & $\begin{array}{c}38,25,33,8,17,16,28,13,23,6,24,2, \\
34,35,26,1,22,9,5,29,21,37,20,3,32, \\
4,10,18,36,30,19\end{array}$ & $\begin{array}{c}38,25,8,33,17,16,28,13,23,6,24,2 \\
34,35,26,22,1,9,5,29,21,37,20,3,32 \\
4,10,18,36,19,30\end{array}$ & $\begin{array}{c}38,25,8,33,17,16,28,13,23,6,24,2 \\
34,35,26,22,1,9,29,5,21,37,20,3,4, \\
32,10,18,36,19,30\end{array}$ \\
\hline
\end{tabular}

The second element of fuzziness concerns the representation of an airport as fuzzy set. In the application discussed in Section 3, only available official data have been used, which are not intrinsically fuzzy as they refer to passengers and movements. In any case, the application to the test case showed that the fuzzy proposed procedure provides results in line with expectations; then specific data surveys can be further realized to test the potentiality of the proposed approach for other clustering goals. Particularly, linguistic variables representing travellers' preferences can be collected to test how users cluster airports from their point of view.

To summarize, the obtained clusters are coherent with the expectations and the fuzzy clustering procedure identifies the airport membership uncertainty by helping planners to better recognize the role of an airport. The two chosen criteria-yearly passengers and movements - in this case lead to similar results in terms of cluster composition and confirm the relationship between carried passengers and number of movements.

Further developments concern the use of some different combined criteria to verify if and how the cluster composition may vary and to verify the use of linguistic variables.

\section{Conflict of Interests}

The authors declare that there is no conflict of interests regarding the publication of this paper.

\section{References}

[1] EUROCONTROL, Trends in Air Traffic. A Place to Stand: Airports in the European Air Network. Volume 3-STATFOR, the EUROCONTROL, Statistics and Forecast Service, Brussels, Belgium, 2007.

[2] European Union, Communication from the Commission Community Guidelines on Financing of Airports and Start-Up Aid to Airlines Departing from Regional Airports, Official Journal of the European Union, Brussels, Belgium, 2005.

[3] M. N. Postorino, "Development of regional airports in EU," in Development of Regional Airports, M. N. Postorino, Ed., pp. 2751, WIT Press (GBR), 2010.

[4] ICAO, Annex 14 Volume I. Aerodrome Design and Operations, 5th edition, 2009.

[5] ICAO, "Ownership, organization and regulatory practices of airports and Air Navigation Service Providers, 2007," Report, documents, 2008. 
[6] V. Adikariwattage, A. G. de Barros, S. C. Wirasinghe, and J. Ruwanpura, "Airport classification criteria based on passenger characteristics and terminal size," Journal of Air Transport Management, vol. 24, pp. 36-41, 2012.

[7] M. A. Madas and K. G. Zografos, "Airport capacity vs. demand: mismatch or mismanagement?" Transportation Research Part A, vol. 42, no. 1, pp. 203-226, 2008.

[8] J. Sarkis and S. Talluri, "Performance based clustering for benchmarking of US airports," Transportation Research Part A, vol. 38, no. 5, pp. 329-346, 2004.

[9] G. Burghouwt and J. Hakfoort, "The evolution of the European aviation network, 1990-1998," Journal of Air Transport Management, vol. 7, no. 5, pp. 311-318, 2001.

[10] P. Malighetti, S. Paleari, and R. Redondi, "Connectivity of the European airport network: "Self-help hubbing" and business implications," Journal of Air Transport Management, vol. 14, no. 2, pp. 53-65, 2008.

[11] M. N. Postorino and F. G. Praticò, "An application of the multicriteria decision-making analysis to a regional multi-airport system," Research in Transportation Business \& Management, vol. 4, pp. 44-52, 2012.

[12] S. Miyamoto, H. Ichihashi, and K. Honda, "Basic methods for cmeans clustering," Studies in Fuzziness and Soft Computing, vol. 229, pp. 9-42, 2008.

[13] L. Bai, J. Liang, C. Dang, and F. Cao, "A novel fuzzy clustering algorithm with between-cluster information for categorical data," Fuzzy Sets and Systems, vol. 215, pp. 55-73, 2013.

[14] A. Ghosh, D. K. Pratihar, M. V. V. Amarnath, G. Dittrich, and J. Mueller, "Fuzzy clustering of mechanisms," Sadhana, vol. 37, no. 5, pp. 539-556, 2012.

[15] Y. H. Kim, S. C. Ahn, and W. H. Kwon, "Computational complexity of general fuzzy logic control and its simplification for a loop controller," Fuzzy Sets and Systems, vol. 111, no. 2, pp. 215224, 2000.

[16] I. Perfilieva and V. Kreinovich, "Fuzzy transform as a new paradigm in fuzzy modeling," Fuzzy Sets and Systems, vol. 180, no. 1, pp. 1-2, 2011.

[17] M. Versaci, S. Calcagno, F. La Foresta, and B. Cammaroto, "Path loss prediction using fuzzy inference system and ellipsoidal rules," American Journal of Applied Sciences, vol. 9, no. 12, pp. 1940-1943, 2012.

[18] R. Redondi, P. Malighetti, and S. Paleari, "Hub competition and travel times in the world-wide airport network," Journal of Transport Geography, vol. 19, no. 6, pp. 1260-1271, 2011.

[19] E. Gegov, M. N. Postorino, M. Atherton, and F. Gobet, "Community structure detection in the evolution of the United States airport network," Advances in Complex Systems, vol. 16, no. 1, 2013.

[20] D. Warnock-Smith and A. Potter, "An exploratory study into airport choice factors for European low-cost airlines," Journal of Air Transport Management, vol. 11, no. 6, pp. 388-392, 2005.

[21] W. K. Li, C. Miyoshi, and R. Pagliari, "Dual-hub network connectivity: an analysis of all Nippon Airways'use of Tokyo's Haneda and Narita airports," Journal of Air Transport Management, vol. 23, pp. 12-16, 2012.

[22] J. T. Rickard, J. Aisbett, G. Gibbon, and D. Morgenthaler, "Fuzzy subsethood for type-n fuzzy sets," in Proceedings of the Annual Meeting of the North American Fuzzy Information Processing Society (NAFIPS '08), New York., NY, USA, May 2008.

[23] J. T. Rickard, J. Aisbett, and G. Gibbon, "Fuzzy subsethood for fuzzy sets of type-2 and generalized type-n," IEEE Transactions on Fuzzy Systems, vol. 17, no. 1, pp. 50-60, 2009.
[24] F. C. Morabito, M. Versaci, G. Pautasso, and C. Tichmann, "Fuzzy-neural approaches to the prediction of disruptions in ASDEX upgrade," Nuclear Fusion, vol. 41, no. 11, pp. 1715-1723, 2001.

[25] B. Kosko, Fuzzy Engineering, Prentice Hall International Editions, 1997.

[26] ENAC, http://www.enac.gov.it/repository/ContentManagement/node/N8911329/Aeroporti_certificati_120927.pdf. 

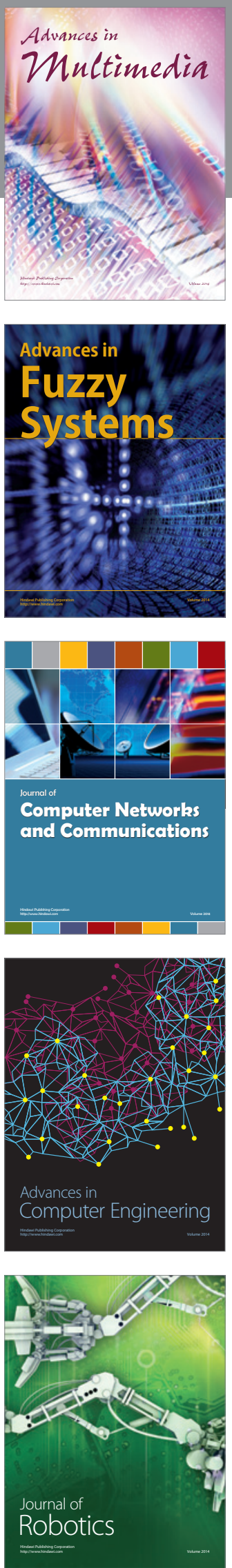

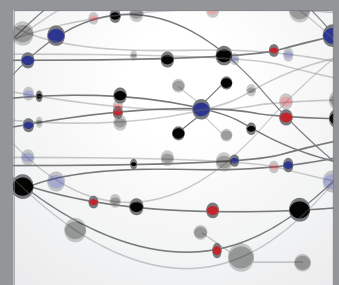

The Scientific World Journal
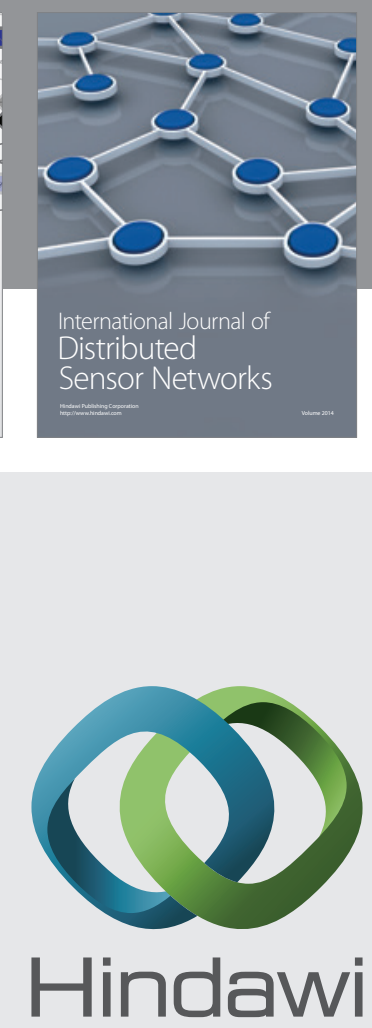

Submit your manuscripts at

http://www.hindawi.com
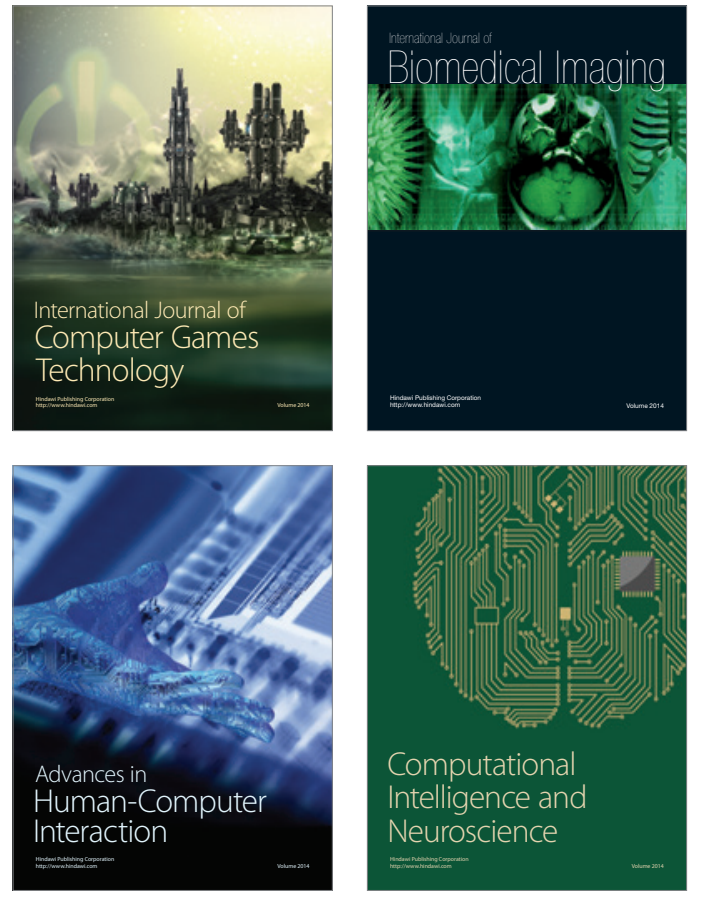
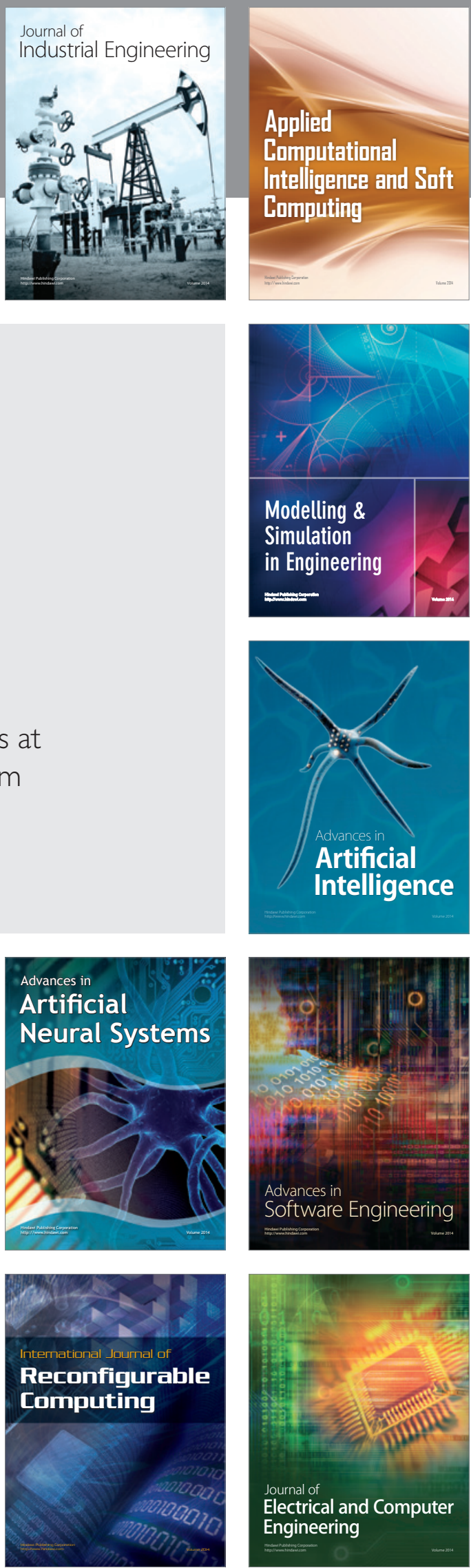